\title{
RENAL DWARFISM
}

\section{A RECORD OF ABNORMALITIES IN CARBOHYDRATE METABOLISM}

\author{
BY \\ M. L. THOMSON, M.A., M.D., M.R.C.P. and VERA K. WILSON, M.Sc. \\ (From the Royal Manchester Children's Hospital)
}

\begin{abstract}
Abnormalities have been observed in the carbohydrate metabolism of three of the four cases of renal dwarfism summarized below. Since the abnormalities do not appear to have been noted in previous cases of renal dwarfism it is felt that a brief record of the findings is of interest.
\end{abstract}

\section{Case summaries}

(Only relevant points are recorded)

Case 1. Male, aged 8 years when first seen. Birth weight, $7 \mathrm{lb}$. Difficult labour lasting three days. Growth had been stunted since the age of 3 years. Thirst and polyuria were noted by the mother at the age of 4 years. When first examined (January, 1942) he showed height, 42 inches; weight, $38 \mathrm{lb}$. He was healthy and intelligent in appearance. The limb-trunk ratio was normal. Blood pressure was $110 / 70 \mathrm{~mm}$. $\mathrm{Hg}$. The clinical examination was negative. The urine was pale, specific gravity 1002 to 1010 , and contained no abnormal constituents. The twenty-four-hour volume was 60 to $110 \mathrm{oz}$. The x-ray of skull showed an unusually massive dorsum sellae and posterior clinoid processes. An excretion urogram (14 c.c. of uropac) showed no dye in pelves, ureters or bladder. Active rickets (type A) was first noted radiologically in November, 1942. The urine was tested daily from January to May, 1942, and for short periods on several subsequent occasions. No albumin found apart from a trace on two occasions. When last seen in November, 1943, however, the urine contained a cloud of albumin and the blood pressure was $155 / 120 \mathrm{~mm}$. $\mathrm{Hg}$ (the first abnormal reading noted). Walking had deteriorated and the rickets had become more severe. He died in March, 1944: no details of death were available.
BloOd Chemistry

(mgm. per cent.)

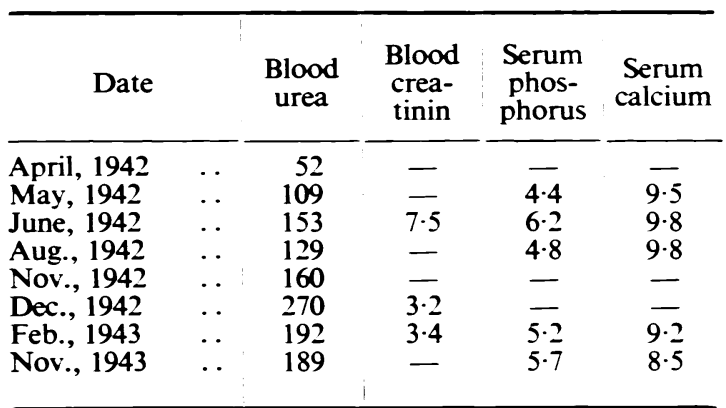

Blood phosphatase activity normal.

Plasma proteins normal.

$\mathrm{CO}_{2}$ combining capacity of plasma 30.9 c.c.

Urea concentration test (April, 1942) $10 \mathrm{gm}$. urea given.

Before urea .. $\quad \ldots \quad \quad \ldots \quad 0 \cdot 3$ per cent.

1 hr. after urea $\quad . \quad \ldots \quad 0.4$, , ,

$\begin{array}{llllll}2 \mathrm{hr} \text {. after urea } & \ldots & \ldots & 0.4 & \text {, , }\end{array}$

3 hr. after urea $\quad . \quad \ldots .0 .5,, \quad$,.

Daily blood sugars while in hospital were within normal limits.

Renal threshold for glucose =approx. $250 \mathrm{mgm}$. per cent. glucose.

INSULIN SENSITIVITY: $20.4 \mathrm{gm}$. glucose by mouth; 3.4 units soluble insulin i.v.

Fasting blood sugar _. $93 \mathrm{mgm}$. per cent.

10 min. after injection . 94 , , , ,

20 min. after injection . 87 , , , ,

30 min. after injection $\quad . \quad 86 \quad$,, $\quad$., , ,.

45 min. after injection $\quad .93 \quad, \quad, \quad, \quad$,

60 min. after injection $\quad . .100,, \quad,, \quad$,

Table 1-Glucose Tolfrance Tests

(mgm. glucose per 100 c.c. of blood)

\begin{tabular}{|c|c|c|c|c|c|c|c|c|c|c|c|c|c|c|c|c|}
\hline Date.. & .. & . & \multicolumn{2}{|c|}{$\begin{array}{c}\text { Feb. 3, } \\
1942\end{array}$} & \multicolumn{2}{|c|}{$\begin{array}{l}\text { Feb. 30, } \\
1942\end{array}$} & \multicolumn{2}{|c|}{$\underset{1942}{\operatorname{Mar} .19}$} & \multicolumn{2}{|c|}{$\begin{array}{c}\text { June } 17, \\
1942\end{array}$} & \multicolumn{2}{|c|}{$\begin{array}{c}\text { July } 23, \\
1942\end{array}$} & \multicolumn{2}{|c|}{$\begin{array}{l}\text { Nov. } 24 \text {, } \\
1942\end{array}$} & \multicolumn{2}{|c|}{$\begin{array}{l}\text { Nov. 10, } \\
1943\end{array}$} \\
\hline Dose .. & . & . & & gm. & & $\mathrm{gm}$. & & gm. & & gm. & & $\mathrm{gm}$. & & gm. & & gm. \\
\hline Fasting blood & sugar & . & 104 & $\begin{array}{c}\text { Urine } \\
\text { sugar } \\
\quad 0\end{array}$ & 92 & $\begin{array}{c}\text { Urine } \\
\text { sugar } \\
0\end{array}$ & 78 & $\begin{array}{c}\text { Urine } \\
\text { sugar } \\
0\end{array}$ & 91 & $\begin{array}{l}\text { Urine } \\
\text { sugar } \\
\quad 0\end{array}$ & 88 & $\begin{array}{c}\text { Urine } \\
\text { sugar } \\
0\end{array}$ & 71 & $\begin{array}{c}\text { Urine } \\
\text { sugar } \\
0\end{array}$ & 99 & $\begin{array}{c}\text { Urine } \\
\text { sugar } \\
0\end{array}$ \\
\hline $\begin{array}{l}\quad \text { Glucos } \\
\frac{1}{2} \text { hr. after } \\
1 \text { hr. after } \\
1 \frac{1}{2} \text { hr. after } \\
2 \text { hr. after } \\
2 \frac{1}{3} \text { hr. after }\end{array}$ & $\begin{array}{l}\text { given } \\
\ldots \\
\ldots \\
\ldots \\
\ldots \\
\ldots\end{array}$ & $\begin{array}{l}\ldots \\
\ldots \\
\ldots \\
\ldots\end{array}$ & $\begin{array}{l}134 \\
202 \\
258 \\
298 \\
174\end{array}$ & $\begin{array}{l}0 \\
0 \\
0 \\
0 \\
0\end{array}$ & $\begin{array}{l}152 \\
243 \\
239 \\
201 \\
205\end{array}$ & $\begin{array}{c}0 \\
0 \\
\text { Trace } \\
\text { Trace } \\
0\end{array}$ & $\begin{array}{l}134 \\
174 \\
179 \\
127 \\
106\end{array}$ & $\begin{array}{l}0 \\
0 \\
0 \\
0 \\
0\end{array}$ & $\begin{array}{r}\overline{169} \\
115 \\
115 \\
70\end{array}$ & $\begin{array}{l}0 \\
0 \\
0 \\
0 \\
0\end{array}$ & $\begin{array}{l}159 \\
207 \\
293 \\
305 \\
286\end{array}$ & $\begin{array}{c}0 \\
0 \\
\text { Trace } \\
- \\
-\end{array}$ & $\begin{array}{l}105 \\
158 \\
221 \\
278 \\
337\end{array}$ & $\begin{array}{l}0 \\
0 \\
0 \\
- \\
-\end{array}$ & $\begin{array}{l}192 \\
309 \\
378 \\
384 \\
378\end{array}$ & $\begin{array}{c}0 \\
\text { Trace } \\
- \\
- \\
-\end{array}$ \\
\hline
\end{tabular}


Case 2. Female, aged 3 years when first seen. Her mother noted the thirst when the patient was just over twelve months old. When first examined (Dec., 1941) she was a backward child. Her height was 31 inches, and weight $23 \mathrm{lb}$. The trunk-limb ratio normal. Her blood pressure was $10570 \mathrm{~mm}$. Hg. The clinical examination was negative. Her urine was pale, specific gravity 1002-1016, and contained no abnormal constituents. The $x$-ray of the skull was normal. No radiological evidence of rickets noted. A plain renal x-ray showed no opaque shadows; kidney shadows not outlined. On normal diet the blood sugar reached its maximum half an hour after mid-day (200 to $230 \mathrm{mgm}$. per cent. of glucose). No sugar was found in urine after blood specimens taken. Daily urine tests over a period of three months and during several short subsequent periods showed no albumin apart from a trace on one occasion. The case was last reviewed in November, 1943. Her height was $34 \frac{1}{2}$ inches and weight $29 \frac{1}{2} \mathrm{lb}$. Her blood pressure was $110 / 70 \mathrm{~mm}$. $\mathrm{Hg}$. The urine contained no albumin. No radiological evidence of rickets traced.

BloOd Chemistry

(mgm. per 100 c.c.)

\begin{tabular}{|c|c|c|c|c|c|}
\hline Date & & $\begin{array}{c}\text { Blood } \\
\text { urea }\end{array}$ & $\begin{array}{l}\text { Blood } \\
\text { crea- } \\
\text { tinin }\end{array}$ & $\begin{array}{c}\text { Serum } \\
\text { calcium }\end{array}$ & $\begin{array}{c}\text { Serum } \\
\text { phos- } \\
\text { phorus }\end{array}$ \\
\hline Jan., 1942 & $\ldots$ & 27 & - & - & - \\
\hline Dec., 1942 & . & 72 & $1 \cdot 5$ & $11 \cdot 5$ & $4 \cdot 7$ \\
\hline May, 1943 & .. & 44 & - & - & - \\
\hline Nov., 1943 & . & 35 & - & $11 \cdot 3$ & $3 \cdot 6$ \\
\hline
\end{tabular}

Blood phosphatase activity normal.

Urea concentration test (Dec., 1942) $4 \mathrm{gm}$. urea given.

$\begin{array}{llllll}\text { Before urea } & \ldots & \ldots & 0.7 & \text { per cent. } \\ 1 \text { hr. after urea } & \ldots & \ldots & 0.9 & . & , \\ 2 \text { hr. after urea } & \ldots & \ldots & 0.5 & ., & , \\ 3 \text { hr. after urea } & \ldots & \ldots & 0.9 & , & ,\end{array}$

Urea clearance $=52$ per cent. average normal function (blood urea $=71 \mathrm{mgm}$. per cent.).

Creatinine clearance, filtration per minute $=78$ c.c.

Insulin sensitivity $=$ normal.

Renal threshold for glucose=approx. $250 \mathrm{mgm}$. glucose. See table 2 for glucose tolerance.

Case 3. Male, aged 7 years. Thirst was first noted when he was twelve months old. He was never very active. Generalized oedema gradually developed in October, 1942. He was treated at another hospital for nephritis and bronchitis for three months during which period the oedema gradually subsided. Thirst and polyuria had been marked since. When first examined (July, 1943) he was a spare, intelligent boy, with sallow skin. His height was 41 inches, weight $34 \mathrm{lb}$. Trunk-limb ratio was normal. His blood pressure was $112,70 \mathrm{~mm}$. $\mathrm{Hg}$. Clinical examination was negative apart from evidence of bronchitis. His urine was pale, specific gravity 1008 to 1016 , and twenty-four-hour volume 14 to $41 \mathrm{oz}$. Albumin (_-) was found. The centrifuged deposit showed 8 red blood cells per field, with occasional pus cells, and a few granular casts. An excretion urogram (12 c.c. uropac) showed no dye in pelves, ureters or bladder. The kidney shadows were small in size. Daily blood sugars half an hour after lunch ranged from 204 to 262 mgm. per cent.

\begin{tabular}{|c|c|c|c|c|}
\hline \multicolumn{5}{|c|}{$\begin{array}{c}\text { BLOOD CHEMISTRY } \\
\text { (mgm. per cent.) }\end{array}$} \\
\hline Date & $\begin{array}{c}\text { Blood } \\
\text { urea }\end{array}$ & $\begin{array}{l}\text { Blood } \\
\text { crea- } \\
\text { tinin }\end{array}$ & $\begin{array}{l}\text { Serum } \\
\text { calcium }\end{array}$ & $\begin{array}{l}\text { Serum } \\
\text { phos- } \\
\text { phorus }\end{array}$ \\
\hline July, 1943 & 165 & $4 \cdot 8$ & $9 \cdot 3$ & $8 \cdot 9$ \\
\hline
\end{tabular}

Urea clearance $=6.5$ per cent. average normal function. (Blood urea $219 \mathrm{mgm}$. per cent.)

Plasma proteins 6.2 gm. per cent.

\section{Glucose Tolerance Test \\ Date: July, 1943}

DOSE.. .. .. $50 \mathrm{gm}$.

\begin{tabular}{llrc} 
Fasting blood sugar & \multicolumn{1}{c}{} & 69 & 0 \\
$\frac{1}{2}$ hr. after glucose & $\ldots$ & 153 & 0 \\
1 hr. after glucose & $\ldots$ & 161 & 0 \\
$1 \frac{1}{2}$ hr. after glucose & $\ldots$ & 193 & 0 \\
2 hr. after glucose & $\ldots$ & 220 & 0 \\
$2 \frac{1}{2}$ hr. after glucose & $\ldots$ & 261 & Trace
\end{tabular}

Renal threshold for glucose=approx. $250 \mathrm{mgm}$. glucose.

Case 4. Female, aged 17 months. She had always been a wet baby, passing urine every quarter hour. There was marked nocturnal enuresis. She drank two pints of cow's milk daily and four cups of tea. On examination her height was 27 inches, weight $14 \mathrm{lb} .8 \mathrm{oz}$. She was able to toddle. The limb-trunk ratio was normal. Clinical examination was negative. Her urine was pale, with a trace of albumin but no other abnormal constituents. There was no radiological evidence of rickets or other bone

Table 2-Glucose Tolerance Tests Case 2:

(mgm. glucose per 100 c.c. of blood)

\begin{tabular}{|c|c|c|c|c|c|c|c|c|c|c|c|c|c|c|}
\hline DATE & . & . & Jan & 1942 & Dec. & 1,1942 & Dec. & 22,1942 & Jan. & 1943 & May & 1943 & Nov & 1943 \\
\hline DOSE & $\cdots$ & .. & & gm. & & gm. & & gm. & & gm. & & gm. & & gm. \\
\hline & & & & Lrine & & Urine & & Crine & & Crine & & Urine & & Urine \\
\hline Fasting blo & igar & .. & 84 & $\begin{array}{l}\text { sugar } \\
0\end{array}$ & 71 & $\begin{array}{c}\text { sugar } \\
0\end{array}$ & - & $\begin{array}{c}\text { sugar } \\
0\end{array}$ & 73 & $\begin{array}{l}\text { sugar } \\
0\end{array}$ & 86 & $\begin{array}{c}\text { sugar } \\
0\end{array}$ & 84 & $\begin{array}{c}\text { sugar } \\
0\end{array}$ \\
\hline$\frac{1}{2} \mathrm{hr}$. after & $\cdots$ & & 121 & 0 & 139 & 0 & 243 & 0 & 172 & 0 & - & 0 & 209 & 0 \\
\hline $1^{2} \mathrm{hr}$. after & . & $\bullet$ & 108 & 0 & 150 & 0 & $\begin{array}{r}300 \\
\text { (child }\end{array}$ & $\begin{array}{c}0 \\
\text { vomited) }\end{array}$ & 224 & 0 & 213 & 0 & 375 & 0 \\
\hline $1 \frac{1}{2} \mathrm{hr}$. after & $\ldots$ & .. & 91 & 0 & 164 & 0 & 224 & 0 & 251 & Trace & 157 & 0 & 175 & 0 \\
\hline $2 \mathrm{hr}$. after & . & $\cdots$ & 87 & 0 & 187 & 0 & 132 & Trace & 197 & 0 & 139 & 0 & 139 & 0 \\
\hline $2 \frac{1}{2}$ hr. after & . & $\cdots$ & - & - & 164 & 0 & 一 & 一 & 143 & 0 & 90 & 0 & 139 & 0 \\
\hline
\end{tabular}




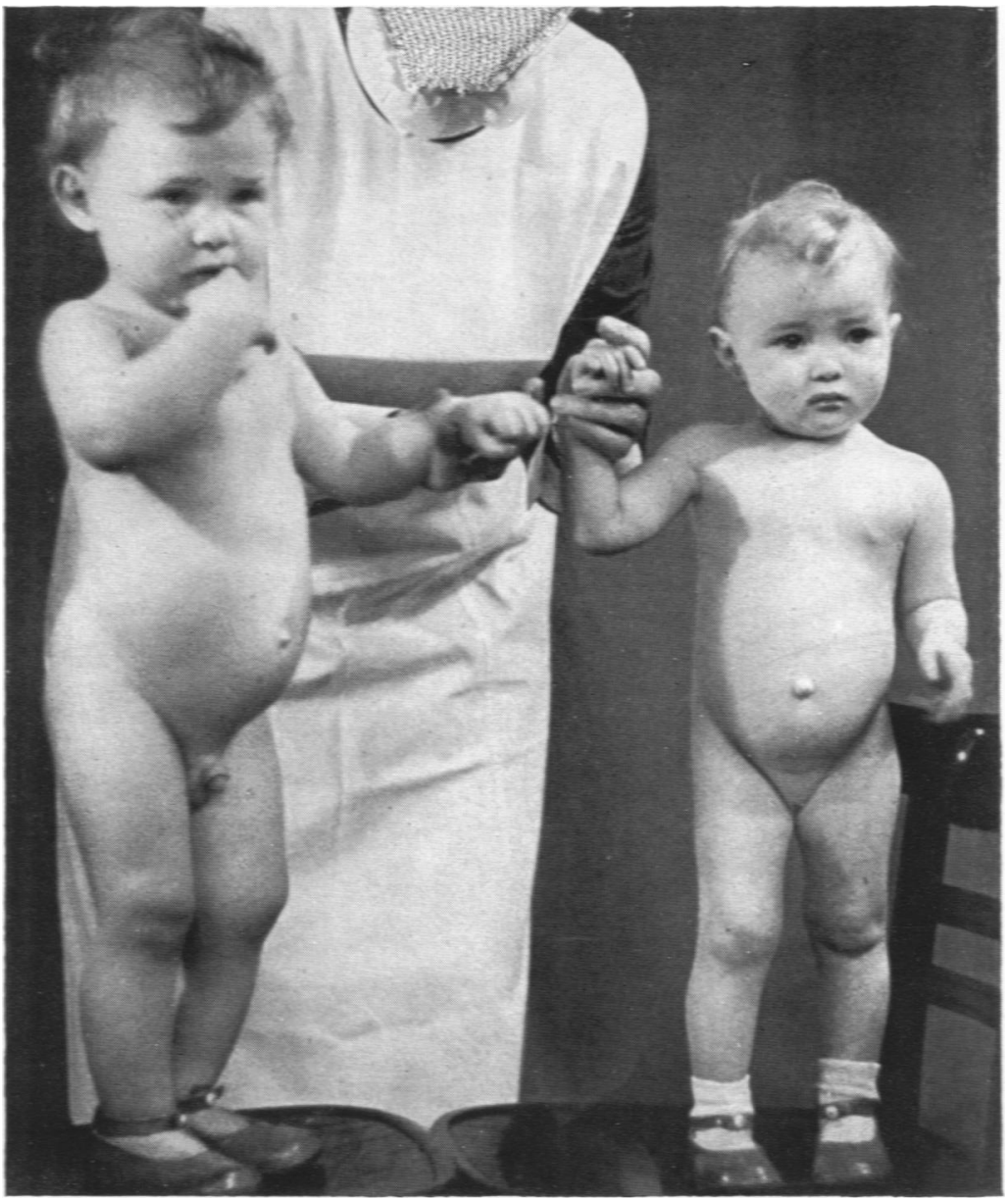

FIG. 1

abnormalities. While in hospital the child drank large quantities of fluid and passed much urine, but it was impossible to estimate even approximately the twenty-four-hour volume. This child has a twin brother who is healthy and normal in every respect (fig. 1).

\section{Blood Chemistry}

(mgm. per cent.)

Date $\quad \begin{gathered}\text { Blood } \\ \text { urea }\end{gathered} \begin{gathered}\text { Serum } \\ \text { calcium }\end{gathered} \quad \begin{gathered}\text { Serum } \\ \text { phosphorus }\end{gathered}$

$\begin{array}{llcc}\text { February, } 1944 & 104 & 13.5 & 5 \cdot 3\end{array}$

Blood phosphatase activity slightly raised.

Plasma proteins $6 \cdot 3 \mathrm{gm}$. per cent.

Glucose Tolerance Tests

\begin{tabular}{|c|c|c|c|c|}
\hline DATE & $\cdots$ & $\cdots$ & Feb. 15,1944 & Mar. 2, 1944 \\
\hline DOSE & $\ldots$ & $\cdots$ & $11 \mathrm{gm}$. & $20 \mathrm{gm}$. \\
\hline \multirow{6}{*}{\multicolumn{2}{|c|}{$\begin{array}{l}\text { Fasting blood sugar } \\
\frac{1}{2} \mathrm{hr} \text {. after glucose } \\
1 \mathrm{hr} \text {. after glucose } \\
1 \frac{1}{2} \mathrm{hr} \text {. after glucose } \\
2 \mathrm{hr} \text {. after glucose } \\
2 \frac{1}{2} \mathrm{hr} \text {. after glucose }\end{array}$}} & $\cdots$ & 91 & - \\
\hline & & $\cdots$ & 128 & 110 \\
\hline & & $\ldots$ & 142 & 135 \\
\hline & & $\ldots$ & 117 & 107 \\
\hline & & $\cdots$ & 75 & - \\
\hline & & $\ldots$ & 71 & - \\
\hline
\end{tabular}

Discussion

The purpose of this article is not to discuss the theories of pathogenesis of renal dwarfism but simply to record the changes in carbohydrate metabolism which have been observed in three of the four cases studied. These cases show a raised renal threshold for sugar (approximately $250 \mathrm{mgm}$. per cent. glucose) and a variable degree of diminished carbohydrate tolerance. From the data available it has not been possible to draw any conclusions regarding the causation of these changes. It is of interest, however, to recall that Lander et al. (1925) obtained similar glucose curves and a raised renal threshold for glucose in adolescents and adults suffering from nephrosclerosis with little or no renal insufficiency and that Castaigne and Chaumerliac (1939) found that a high renal threshold for glucose is very common in chronic sclerosing nephritis.

Hagedorn and Jensen method was used for all blood sugar estimations. (Capillary blood specimens were taken.)

Thanks are due to Dr. H. T. Ashby for allowing the publication of case 2 and to Dr. M. Flowerday for her assistance with the first two cases.

\section{ReFERENCES}

Lander, G. C., Hiller, A., and van Slyke, D. D. (1925). J. clin. Invest., 1, 247.

Castaigne, J., and Chaumerliac, J. (1939). Nutrition, 9, 19. 